\title{
Tuned Lamb Wave Excitation and Detection with Piezoelectric Wafer Active Sensors for Structural Health Monitoring
}

\author{
VICTOR GIURGIUTIU* \\ Mechanical Engineering Department, University of South Carolina, Columbia, SC 29208
}

\begin{abstract}
The capability of embedded piezoelectric wafer active sensors (PWAS) to excite and detect tuned Lamb waves for structural health monitoring is explored. First, a brief review of Lamb waves theory is presented. Second, the PWAS operating principles and their structural coupling through a thin adhesive layer are analyzed. Then, a model of the Lamb waves tuning mechanism with PWAS transducers is described. The model uses the space domain Fourier transform. The analysis is performed in the wavenumber space. The inverse Fourier transform is used to return into the physical space. The integrals are evaluated with the residues theorem. A general solution is obtained for a generic expression of the interface shear stress distribution. The general solution is reduced to a closed-form expression for the case of ideal bonding which admits a closed-form Fourier transform of the interfacial shear stress. It is shown that the strain wave response varies like $\sin \gamma a$, whereas the displacement response varies like sinc $\gamma a$. Maximum coupling is achieved when the PWAS length equals the half wavelength of a particular Lamb wave mode. Since Lamb wave modes wavelengths vary with frequency, the tuning of certain modes at certain frequencies can thus be achieved. Tuning curves are derived and verified against experimental results. A particular $S_{0}$ mode 'sweet spot' is found at $300 \mathrm{kHz}$ for a $7-\mathrm{mm}$ PWAS attached to a 1.6-mm aluminum plate. Crack detection via the pulse echo technique using the phased array principle and tuned $S_{0}$ mode Lamb waves is demonstrated as an effective structural health monitoring method.
\end{abstract}

Key Words: structural health monitoring, Lamb waves, piezoelectric wafer active sensors, aging aircraft, cracks, damage, faults, diagnostics, prognostics, PWAS, SHM.

\section{INTRODUCTION}

$\mathbf{S}$ TRUCTURAL health monitoring (SHM) addresses an urgent need of our aging infrastructure. The United States spends many billions of dollars every year on the maintenance of plant equipment and facilities. Maintenance and repairs represent about a quarter of the commercial aircraft operating costs. Much of the civilian and military aircraft fleets have exceeded their design life. Some of NASA's space shuttles are more than 20 years old. The mounting costs of maintaining our aging infrastructure can be addressed through SHM systems that will reduce scheduled and unscheduled repairs while increasing safety and reliability. Boeing's new 7E7 aircraft will have a full-time built-in SHM system consisting of sensors embedded in the structure to assess the state of structural health (ButterworthHayes, 2003).

SHM systems consist of networks of embedded sensors that are permanently inserted into the structure and monitored over time. SHM can be either passive

*E-mail: victorg@sc.edu or active. Passive SHM infers the state of the structure using passive sensors (loading, stress, environmental condition, performance indicators, acoustic emission from cracks, etc.) that are monitored over time and fed back into a structural model. Passive SHM only 'listens' to the structure but does not interact with it. Active SHM is different from passive SHM because it uses sensors to interrogate the structure in order to detect damage presence, extent, and intensity. Active sensors act upon the structure in much the same way that conventional nondestructive evaluation (NDE) transducers do. Unlike passive sensors (which only listen to the structure), active sensors interact directly with the structure and find its state of health and reliability. One way to achieve structural interrogation is through ultrasonic waves. Among ultrasonic waves, the Lamb waves are particularly advantageous because they can propagate over large distances in plates and shells (Rose 1995, 2002; Krautkramer, 1998; Seale et al., 1998; Alleyne et al., 2001; Dalton et al., 2001; Thomson and Chimenti, 2002). Active SHM systems using interrogative Lamb waves would be able to cover large areas from one single location. Such systems would be 
cost-effective and efficient. However, conventional ultrasonic transducers are inappropriate for SHM applications due to their cost, weight, and size. Conventional ultrasonic transducers could not be embedded in large numbers into a structure without incurring important cost and weight penalties. For SHM applications, new types of Lamb wave transducers must be developed; they must be small, lightweight, unobtrusive, and low cost.

\section{LAMB WAVES}

Lamb waves, a.k.a. guided plate waves, are a type of ultrasonic waves that remain guided between two parallel free surfaces, such as the upper and lower surfaces of a plate or shell. Lamb wave theory is fully documented in a number of textbooks (Viktorov, 1967; Graff, 1975; Achenbach, 1999; Rose, 1999). Here, we only reproduce the bare essentials. The analysis starts from the wave equations

$$
\begin{aligned}
& \frac{\partial^{2} \phi}{\partial x^{2}}+\frac{\partial^{2} \phi}{\partial y^{2}}+\frac{\omega^{2}}{c_{L}^{2}} \phi=0 \\
& \frac{\partial^{2} \psi}{\partial x^{2}}+\frac{\partial^{2} \psi}{\partial y^{2}}+\frac{\omega^{2}}{c_{T}^{2}} \psi=0
\end{aligned}
$$

where $\phi$ and $\psi$ are two potential functions, $c_{P}^{2}=(\lambda+2 \mu) / \rho$ and $c_{S}^{2}=\mu / \rho$ are the pressure (longitudinal) and shear (transverse) wavespeeds, $\lambda$ and $\mu$ are the Lame constants, and $\rho$ is the mass density. The time dependence is assumed harmonic in the form $\mathrm{e}^{-i \omega t}$. The general solution of Equation (1) is

$$
\begin{aligned}
& \phi=\left(A_{1} \sin p y+A_{2} \cos p y\right) e^{i(\xi x-\omega t)} \\
& \psi=\left(B_{1} \sin q y+B_{2} \cos q y\right) e^{i(\xi x-\omega t)}
\end{aligned}
$$

where $\xi=\omega / c$ is the wavenumber and

$$
p^{2}=\frac{\omega^{2}}{c_{L}^{2}}-\xi^{2}, \quad q^{2}=\frac{\omega^{2}}{c_{T}^{2}}-\xi^{2}
$$

The four integration constants, $A_{1}, A_{2}, B_{1}, B_{2}$, are to be found from the boundary conditions. Using the relations between the potential functions and the displacements, stresses, and strains

$$
\begin{aligned}
& u_{x}=\frac{\partial \phi}{\partial x}+\frac{\partial \psi}{\partial y}, \quad \tau_{y x}=\mu\left(2 \frac{\partial^{2} \phi}{\partial x \partial y}-\frac{\partial^{2} \psi}{\partial x^{2}}+\frac{\partial^{2} \psi}{\partial y^{2}}\right) \\
& u_{y}=\frac{\partial \phi}{\partial y}-\frac{\partial \psi}{\partial x}, \quad \tau_{y y}=\lambda\left(\frac{\partial^{2} \phi}{\partial x^{2}}+\frac{\partial^{2} \phi}{\partial y^{2}}\right)+2 \mu\left(\frac{\partial^{2} \phi}{\partial x^{2}}-\frac{\partial^{2} \psi}{\partial x \partial y}\right) \\
& \varepsilon_{x}=\frac{\partial u_{x}}{\partial x}
\end{aligned}
$$

we obtain

$$
\begin{aligned}
u_{x}=[ & \left(A_{2} i \xi \cos p y+B_{1} q \cos q y\right) \\
& \left.+\left(A_{1} i \xi \sin p y-B_{2} q \sin q y\right)\right] e^{i(\xi x-\omega t)} \\
u_{y}=[ & -\left(A_{2} p \sin p y+B_{1} i \xi \sin q y\right) \\
& \left.+\left(A_{1} p \cos p y-B_{2} i \xi \cos q y\right)\right] e^{i(\xi x-\omega t)}
\end{aligned}
$$

The terms in Equation (5) have been grouped into two parts, the first corresponding to symmetric motion and the second to antisymmetric motion (Figure 1).

For free wave motion, we derive the homogenous solution by applying the stress-free boundary conditions at the upper and lower surfaces $(y= \pm d$, where $d$ is the plate half thickness). One obtains the characteristic equations (Achenbach, 1999):

$$
\begin{aligned}
D_{S}= & \left(\xi^{2}-q^{2}\right)^{2} \cos p d \sin q d+4 \xi^{2} p q \sin p d \cos q d=0 \\
& \quad(\text { symmetric motion) } \\
D_{A}= & \left(\xi^{2}-q^{2}\right)^{2} \sin p d \cos q d+4 \xi^{2} p q \cos p d \sin q d=0 \\
& \quad \text { (antisymmetric motion) }
\end{aligned}
$$

Equations (6) and (7) can be rewritten in the more compact form as the Rayleigh-Lamb equation:

$$
\frac{\tan p d}{\tan q d}=-\left[\frac{4 \xi^{2} p q}{\left(\xi^{2}-q^{2}\right)^{2}}\right]^{ \pm 1}
$$

where +1 corresponds to symmetric $(S)$ motion and -1 to antisymmetric $(A)$ motion. Equations (6) and (7) accept a number of eigenvalues, $\xi_{0}^{S}, \xi_{1}^{S}, \xi_{2}^{S}, \ldots$, and $\xi_{0}^{A}, \xi_{1}^{A}, \xi_{2}^{A}, \ldots$, respectively. To each eigenvalue corresponds a set of eigencoefficients: $\left(A_{2}, B_{1}\right)$ for the symmetric case, and $\left(A_{1}, B_{2}\right)$ for the antisymmetric case. Substitution of these coefficients into Equation (5) yields the corresponding Lamb mode shapes. The symmetric modes are designated $S_{0}, S_{1}, S_{2}, \ldots$, while the antisymmetric are designated $\mathrm{A}_{0}, \mathrm{~A}_{1}, \mathrm{~A}_{2}, \ldots$

Since the coefficients $p$ and $q$ in Equations (6) and (7) depend on the angular frequency $\omega$, the eigenvalues $\xi_{i}^{S}$ and $\xi_{i}^{A}$ will change with the excitation frequency. The corresponding wavespeeds, given by $c_{i}=\omega / \xi_{i}$, will also change with frequency. The change of wavespeed with frequency produces wave dispersion. Lamb waves are highly dispersive, and their speed depends on the

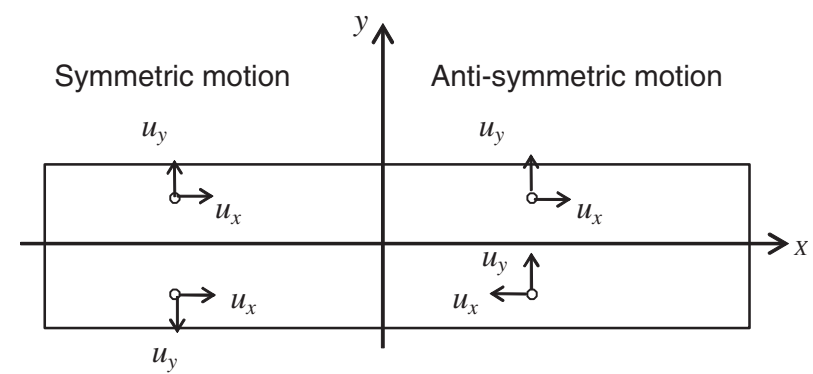

Figure 1. Symmetric and antisymmetric particle motion across the plate thickness. 
product $f d$ between the frequency, $f$, and the plate halfthickness, $d$. At a given frequency thickness product, for each solution of the Rayleigh-Lamb equation, one finds a corresponding Lamb wavespeed and a corresponding Lamb wave mode. The plot of the Lamb wavespeeds against the $f d$ product gives the wavespeed dispersion curves (Figure 2). Figure 3 presents the displacement fields across the thickness for various Lamb modes at various frequencies. It is apparent that the bow and number of nodes of the Lamb wave mode across the thickness increases with frequency and mode number. At low frequencies, the symmetric $S_{0}$ Lamb wave mode is almost straight across the thickness and resembles the displacement field of the simple axial wave. Similarly, the antisymmetric $A_{0}$ Lamb wave mode resembles the displacement field of the simple flexural wave. Another important fact to be noticed is that the Lamb waves have the dual characteristic of being standing waves across the thickness and traveling wave along the plate. For illustration, Figure 4 shows typical displacement fields of the $S_{0}$ and $A_{0}$ Lamb wave modes in both $x$ - and $y$-directions.

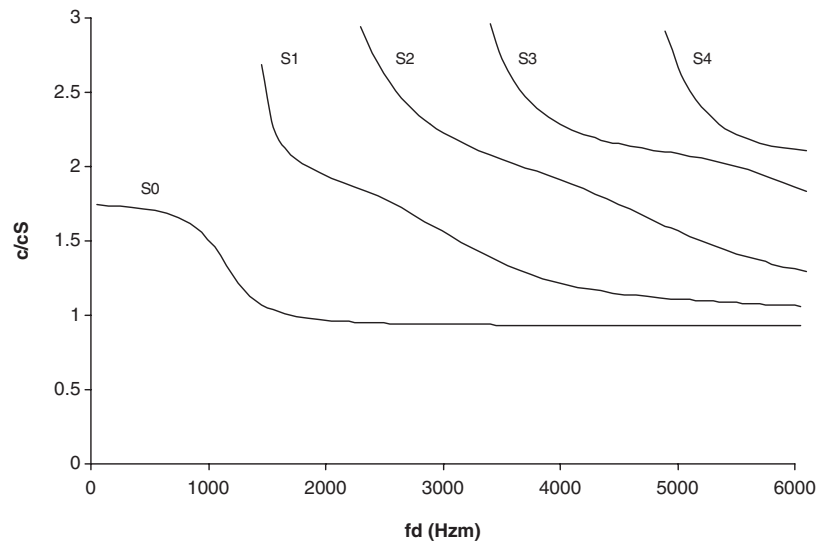

(a)

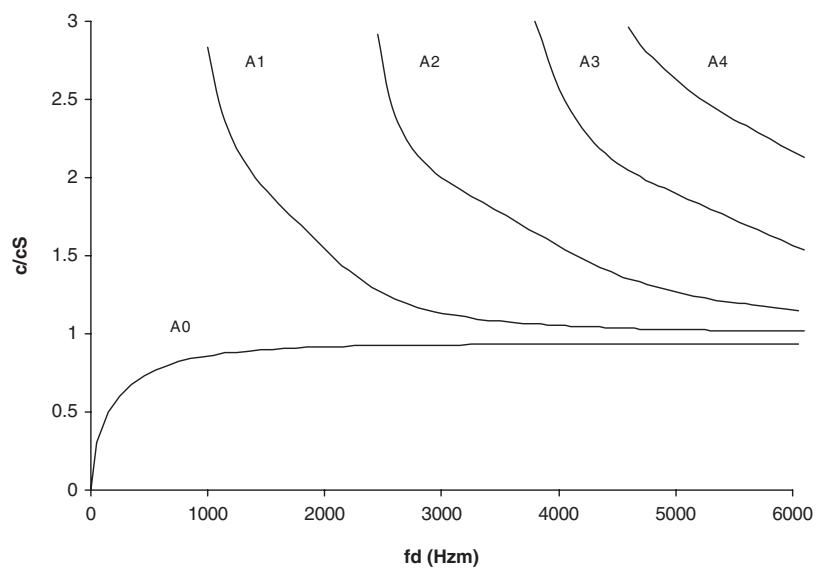

(b)

Figure 2. Wavespeed dispersion curves for Lamb waves in an aluminum plate $\left(c_{s}=\right.$ shear wavespeed, $d=$ half-thickness of the plate): (a) symmetric modes and (b) antisymmetric modes.

\section{PIEZOELECTRIC WAFER ACTIVE SENSORS}

In recent years, several investigators (Chang, 1995, 1998; Keilers and Chang, 1995; Agrawal, 1996; Moulin et al., 1997; Wang and Chang, 2000; Dupont et al., 2001; Lin and Yuan, 2001a,b; Diamandi et al., 2002; DiazValdes and Soutis, 2002; Ihn and Chang, 2002; Giugiutiu and Zagrai, 2000; Giugiutiu et al., 2002;) have explored the generation and detection of structural waves with piezoelectric wafer active sensors (PWAS). Most of the methods used in conventional NDE, such as pitch-catch, pulse echo, and phased arrays, have also been demonstrated experimentally with PWAS (Giurgiutiu et al., 2002, 2003). These successful experiments have positioned PWAS as an enabling technology for the development and implementation of active SHM systems. PWAS are inexpensive, nonintrusive, unobtrusive, and minimally invasive. They can be surface mounted on existing structures, inserted between the layers of lap joints, or placed inside composite materials. Figure 5 shows an array of $7 \mathrm{~mm}$ square PWAS mounted on an aircraft panel, adjacent to rivet heads and to an electric discharge machined (EDM) simulated crack. The minimally invasive nature of the PWAS devices is apparent. A PWAS weighs $0.068 \mathrm{~g}$, is $0.2 \mathrm{~mm}$ thick, and costs around $\$ 10$ each. In contrast, a conventional ultrasonic transducer weighs $50 \mathrm{~g}$, is $20 \mathrm{~mm}$ thick, and costs around $\$ 300$.

\section{PWAS Operating Principles}

PWAS operate on the piezoelectric principle and achieve direct transduction of electric energy into elastic energy and vice versa. Their constitutive equations couple the electrical and mechanical variables in the material (mechanical strain, $S_{i j}$, mechanical stress, $T_{k l}$, electrical field, $E_{k}$, and electrical displacement $D_{j}$ ) in the form:

$$
\begin{aligned}
& S_{i j}=s_{i j k l}^{E} T_{k l}+d_{k i j} E_{k} \\
& D_{j}=d_{j k l} T_{k l}+\varepsilon_{j k}^{T} E_{k},
\end{aligned}
$$

where $s_{i j k l}^{E}$ is the mechanical compliance of the material measured at zero electric field $(E=0), \varepsilon_{j k}^{T}$ is the dielectric permittivity measured at zero mechanical stress $(T=0)$, and $d_{k i j}$ represents the piezoelectric coupling effect. An alternating voltage applied to the PWAS terminals produces an oscillatory expansion and contraction of the PWAS material. Vice versa, an oscillatory expansion and contraction of the PWAS material produces an alternating voltage at the PWAS terminals. In Lamb waves applications, PWAS couple their in-plane motion, excited through the piezoelectric effect, with the Lamb waves particle motion on the material surface. They can be both exciters and 

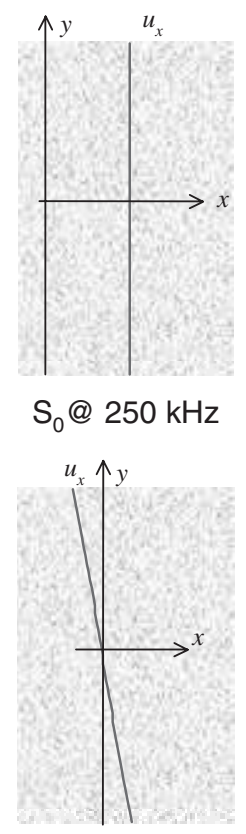

$\mathrm{A}_{0} @ 250 \mathrm{kHz}$

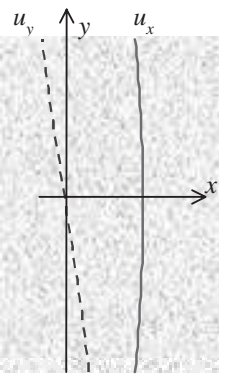

$\mathrm{S}_{0} @ 1000 \mathrm{kHz}$

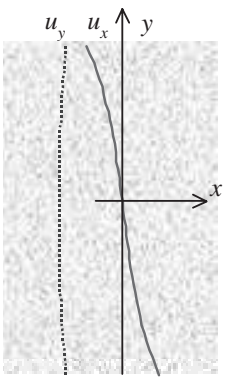

$\mathrm{A}_{0} @ 1000 \mathrm{kHz}$

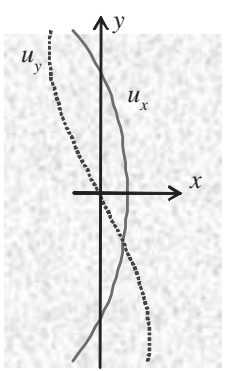

$\mathrm{S}_{0} @ 3000 \mathrm{kHz}$

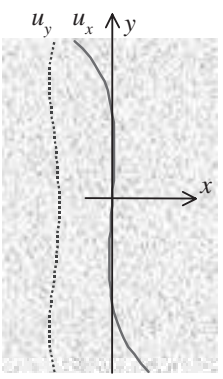

$\mathrm{A}_{0} @ 3000 \mathrm{kHz}$

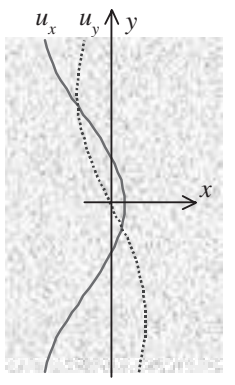

$\mathrm{S}_{1} @ 3000 \mathrm{kHz}$

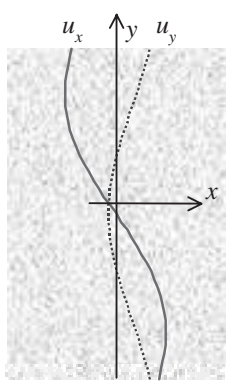

$A_{1} @ 3000 \mathrm{kHz}$

Figure 3. Displacement fields across the thickness for various Lamb modes at various frequencies showing how Lamb mode curvature increases with frequency and mode number.

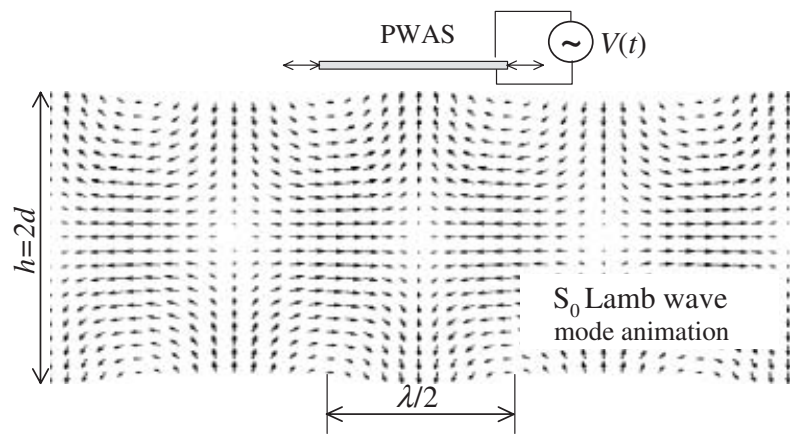

(a)

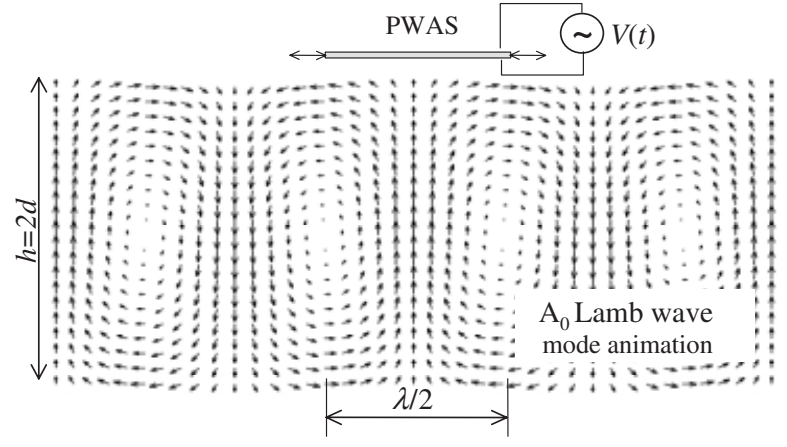

(b)

Figure 4. PWAS interaction with Lamb modes: (a) symmetric Lamb mode $S_{0}$ and (b) antisymmetric Lamb mode $A_{0}$.

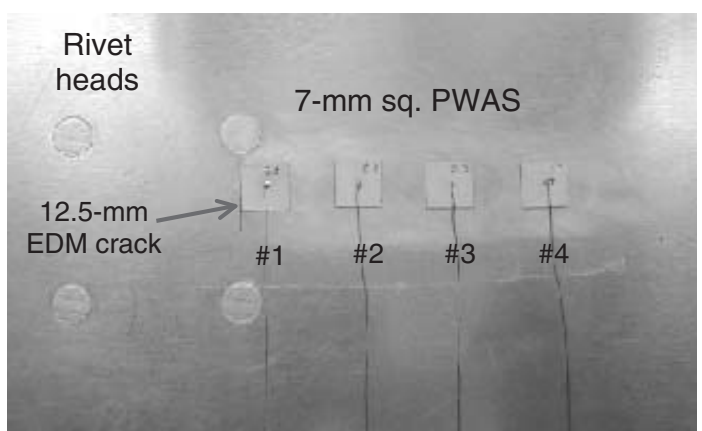

Figure 5. Piezoelectric wafer active sensors (PWAS) mounted on aircraft panel.

detectors of elastic Lamb waves traveling in the material. Though both PWAS and conventional ultrasonic transducers operate on the piezoelectric effect, their modes of operation are in fact quite different:

(1) Conventional ultrasonic transducers are weakly coupled with the investigated structure through gel, water, or air. In contrast, PWAS are strongly coupled with the structure through an adhesive bond.

(2) Conventional ultrasonic transducers are resonant narrowband devices. In contrast, PWAS are nonresonant broadband devices that can be tuned selectively into certain Lamb modes.

(3) Conventional ultrasonic transducers excite and sense the Lamb waves in the structure indirectly through acoustic waves impinging on the structural surface and the mode conversion phenomenon. In contrast, PWAS excite and sense the Lamb waves in the structure directly through in-plane strain coupling. 
Conventional ultrasonic transducers have been studied extensively. Their behavior is modeled and understood relatively well (Silk, 1984). In contrast, the modeling and understanding of the PWAS transducers and their Lamb wave interaction with the host structure is only at the beginning. To date, most of the progress in utilizing the PWAS technology for active SHM has been achieved empirically, through successful proof of concept demonstrations. Some modeling studies have also been conducted, but most have focused on the wave propagation in the structure (Kim et al., 1997; Lin and Yuan, 2001b; Lee and Staszewski, 2003). A comprehensive modeling of the interaction between the PWAS, the structure, and the Lamb waves traveling at ultrasonic frequencies through the structure is needed as an essential design tool.

\section{Shear Layer Coupling between the PWAS and the Structure}

Consider a surface mounted PWAS and the adjacent structure. A typical micrograph of such a situation is shown in Figure 6(a). Shown in this micrograph are $0.2-\mathrm{mm}$ thick PWAS and the 1-mm thick structural substrate. Also shown is the thin bond line connecting the PWAS and the structure. The roughness on the upper and lower PWAS surfaces represents the $\mathrm{Ni}$ electrodes which were deposited onto the PWAS through a sputtering technique. The associated model assumes:

(a) PWAS of length $l_{a}=2 a$, thickness $t_{a}$, and elastic modulus $E_{a}$

(b) bonding layer of thickness $t_{b}$, and shear modulus $G_{b}$

(c) structure of thickness $t=2 d$, and elastic modulus $E$

The connection between the PWAS and the structure is achieved through the interfacial shear stress, $\tau(x, t)=\tau_{a}(x) e^{i \omega t}$. The shear stress intensity and distribution depend on the relative deformation of the PWAS and the structure. For static deformation and quasi-static low frequency vibrations, Crawley et al. $(1987,1990)$ developed a 1-D plane strain analysis using the Euler-Bernoulli hypothesis across the plate thickness, i.e., uniform displacement for axial motion, and linear displacement for flexural motion. The resulting shear transfer solution is written in terms of hyperbolic functions, i.e.,

$$
\tau_{0}(x)=\frac{t_{a}}{a} \frac{\psi}{\alpha+\psi} E_{a} \varepsilon_{I S A}\left(\Gamma a \frac{\sinh \Gamma x}{\cosh \Gamma a}\right)
$$

(Interfacial shear stress in the bond layer)

where,

$$
\psi=\frac{E t}{E_{a} t_{a}} \quad \text { and } \quad \Gamma^{2}=\frac{G_{b}}{E_{a}} \frac{1}{t_{a} t_{b}} \frac{\alpha+\psi}{\psi} .
$$

The parameter $\alpha$ depends on the stress and strain distribution across the structural thickness. For lowfrequency coupled axial-bending motion under the Euler-Bernoulli hypothesis, this parameter takes the value $\alpha=4$. However, this value is expected to change as frequency increases and the Lamb modes gain curvature and nodal points across the thickness.

The shear transfer along the PWAS is controlled by the product between the shear lag parameter, $\Gamma$, and the PWAS half-length, $a$. For low values of the $\Gamma a$ product, the shear transfer is distributed along the PWAS length, and the shear stresses have a relatively low intensity. For high values of the $\Gamma a$ product the shear transfer is localized towards the PWAS ends, and the shear stresses have high intensity. Figure 7 presents numerical results corresponding to an APC-850 PWAS $\left(E_{a}=63 \mathrm{GPa}, t_{a}=0.2 \mathrm{~mm}, l_{a}=7 \mathrm{~mm}, d_{31}=-175 \mathrm{~mm} /\right.$ $\mathrm{kV}$ ) attached to a thin-wall aluminum structure $(E=70 \mathrm{GPa}$ and $t=1 \mathrm{~mm})$ through a bond layer of $G_{b}=2 \mathrm{GPa}$. The bond layer thickness was allowed to vary from 1 to $100 \mu \mathrm{m}$. It is apparent that a relatively thick bonding layer produces a slow transfer over the entire span of the PWAS (the ' $100 \mu \mathrm{m}$ ' curves in Figure 7), whereas a thin bonding layer produces a
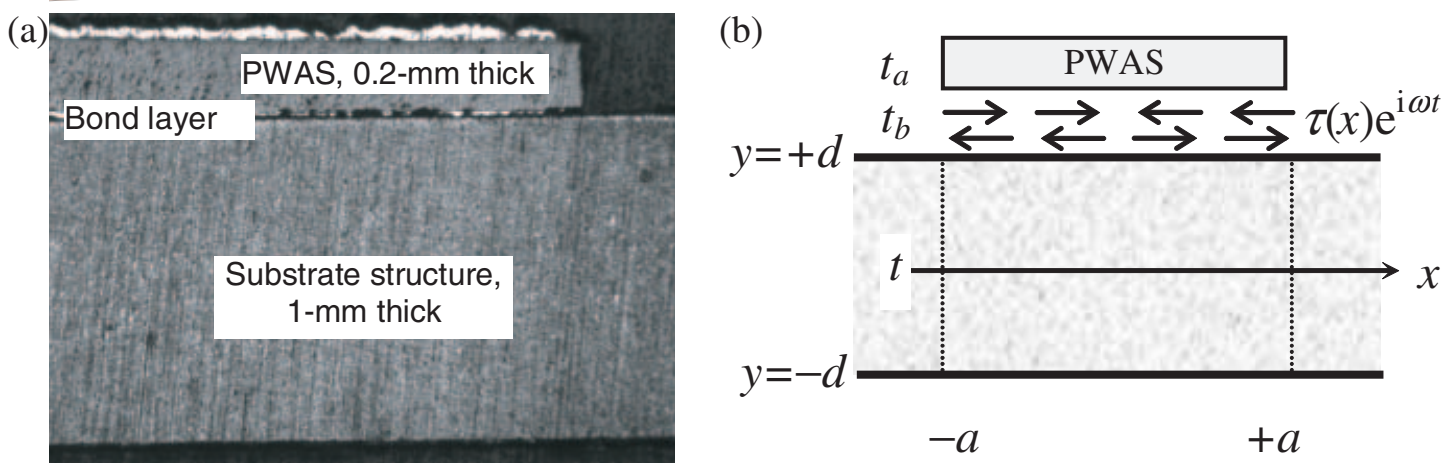

Figure 6. Shear layer interaction between the PWAS and the structure: (a) micrograph and (b) modeling. 


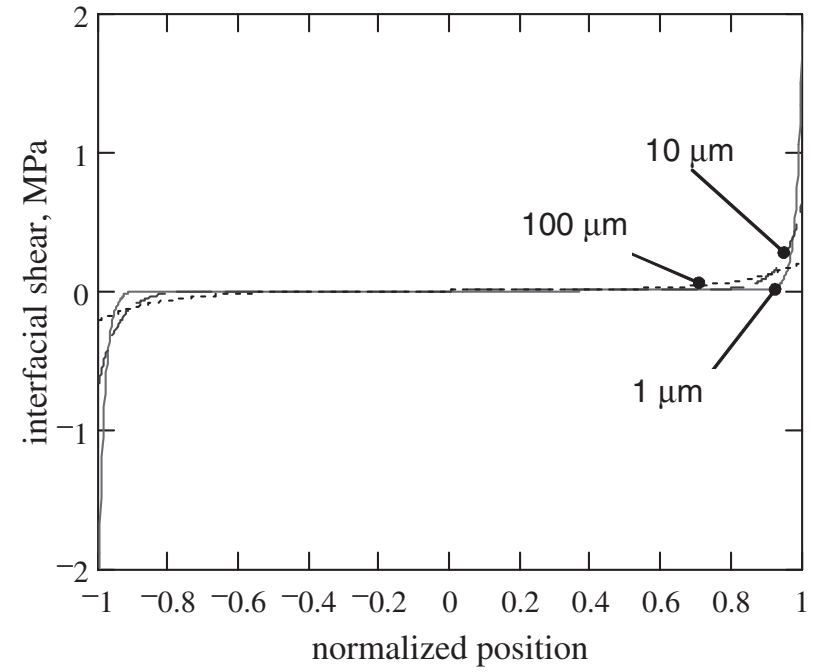

Figure 7. Variation of shear lag transfer mechanism with bond thickness for a APC-850 PWAS $\left(E_{a}=63 \mathrm{GPa}, t_{a}=0.2 \mathrm{~mm}, \mathrm{l}_{a}=7 \mathrm{~mm}\right.$, $d_{31}=-175 \mathrm{~mm} / \mathrm{kV}$ ) attached to a thin-wall aluminum structure $(E=70 \mathrm{GPa}$ and $t=1 \mathrm{~mm})$ through a bond layer of $\mathrm{G}_{b}=2 \mathrm{GPa}$.

very rapid transfer (the ' $1 \mu \mathrm{m}$ ' curves in Figure 7 ). As the bond layer becomes thinner and stiffer, the shear stress becomes more and more confined to the PWAS ends. In the case of ideal bonding we recover the pin force model, in which all the load transfer takes place over an infinitesimal region at the PWAS ends. In this case, the shear stress distribution along the PWAS interface can be expressed using $\delta(x)$ Dirac function, i.e.,

$$
\tau_{a}(x)=a \tau_{0}[\delta(x-a)-\delta(x+a)]
$$

where $a \tau_{0}$ is the pin force applied at PWAS ends.

\section{LAMB WAVE MODES TUNING WITH PWAS TRANSDUCERS}

It has been already mentioned that an alternating voltage applied to the PWAS terminals produces an oscillatory expansion and contraction of the PWAS material. Vice versa, an oscillatory expansion and contraction of the PWAS material produces an alternating voltage at the PWAS terminals. In Lamb wave applications, PWAS couple their in-plane motion, excited through the piezoelectric effect, with the Lamb waves in-plane strain on the material surface. Thus, PWAS can be both exciters and detectors of elastic Lamb waves traveling in the material. In this section, we analyze the case of a surface mounted PWAS (Figure 6). Because the PWAS is mounted asymmetric with respect to the midthickness axis, both symmetric and antisymmetric Lamb wave modes can be excited and detected. We will show that conditions can be found for which the PWAS will couple preferentially with only one of these Lamb wave modes, i.e., the PWAS is tuned with that particular Lamb wave mode. The modeling of this phenomenon is done as follows.

Consider the surface-mounted PWAS shown in Figure 6(b). The PWAS is excited electrically with a time-harmonic voltage $\mathrm{Ve}^{-\mathrm{i} \omega t}$. As a result, the PWAS expands and contracts, and a time harmonic interfacial shear stress, $\tau_{\alpha}(x) \mathrm{e}^{-\mathrm{i} \omega t}$, develops between the PWAS to the structure. This shear stress acts on the structure over the interval $x \in(-a,+a)$. Thus, the $\tau_{y x}$ boundary condition on the structure upper surface $(y=d)$ is given by:

$$
\left.\tau_{y x}\right|_{y=h}=\tau_{a}(x)=\tau_{0}(x)[H(x+a)-H(x-a)]
$$

where $H(x)$ is the Heaviside step function and the timeharmonic variation $\mathrm{e}^{-\mathrm{i} \omega t}$ is implied. From this point onwards, the analysis is performed in the wavenumber domain by applying the space domain Fourier transform:

$$
\tilde{f}(\xi)=\int_{-\infty}^{\infty} f(x) e^{-i \xi x} d x, \quad f(x)=\frac{1}{2 \pi} \int_{-\infty}^{\infty} \tilde{f}(\xi) e^{i \xi x} d \xi
$$

The space domain Fourier transform of the excitation (12) is

$$
\tilde{\tau}_{a}=\int_{-\infty}^{\infty} \tau_{0}(x)[H(x+a)-H(x-a)] e^{-i \xi x} d x
$$

Applying the space domain Fourier transform to the wave equations (1) and the displacements, stresses, and strains, we obtain:

$$
\begin{aligned}
& \frac{d^{2} \tilde{\phi}}{d y^{2}}+p^{2} \tilde{\phi}=0 \\
& \frac{d^{2} \tilde{\psi}}{d y^{2}}+q^{2} \tilde{\psi}=0
\end{aligned}
$$

$$
\begin{array}{rlrl}
\tilde{u}_{x}=i \xi \tilde{\phi}+\frac{d \tilde{\psi}}{d y} & \tilde{\tau}_{y x} & =\mu\left(2 i \xi \frac{d \tilde{\phi}}{d y}+\xi^{2} \tilde{\psi}+\frac{\partial^{2} \tilde{\psi}}{\partial y^{2}}\right) \\
\tilde{u}_{y}=\frac{d \tilde{\phi}}{d y}-i \xi \tilde{\psi} & \tilde{\tau}_{y y} & =\lambda\left(-\xi^{2} \tilde{\phi}+\frac{d^{2} \tilde{\phi}}{d y^{2}}\right) \\
& +2 \mu\left(-\xi^{2} \tilde{\phi}-i \xi \frac{d \tilde{\psi}}{d y}\right) \\
\tilde{\varepsilon}_{x}=i \xi u_{x} &
\end{array}
$$

where, $p$ and $q$ are given by Equation (3). Equation (15) accepts the general solution

$$
\begin{aligned}
& \tilde{\phi}=A_{1} \sin p y+A_{2} \cos p y \\
& \tilde{\psi}=B_{1} \sin q y+B_{2} \cos q y
\end{aligned}
$$



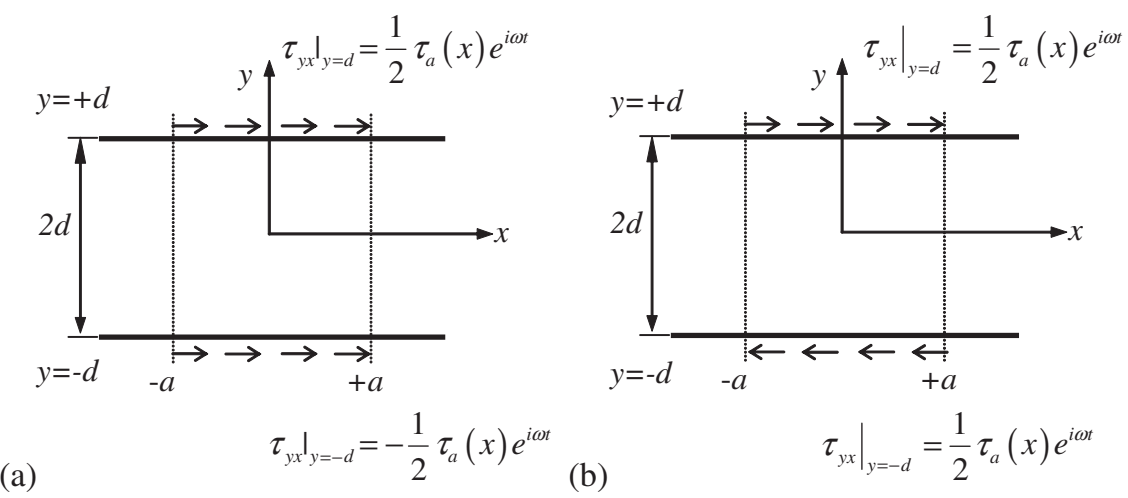

Figure 8. Symmetric and antisymmetric loading from a PWAS of width 2a mounted on the plate upper surface: (a) symmetric loading and (b) antisymmetric loading.

As before, the constants $A_{2}$ and $B_{1}$ correspond to symmetric motion, while $A_{1}$ and $B_{2}$ correspond to antisymmetric motion. The excitation can be also split into symmetric and antisymmetric components (Figure 8), i.e.,

$$
\begin{aligned}
& \left.\tilde{\tau}\right|_{y=d}=\left.\frac{1}{2} \tilde{\tau}_{a}\right|_{y=d}+\left.\frac{1}{2} \tilde{\tau}_{a}\right|_{y=d}=\tilde{\tau}_{a} \quad \text { upper surface } \\
& \left.\tilde{\tau}\right|_{y=-d}=-\left.\frac{1}{2} \tilde{\tau}_{a}\right|_{y=-d}+\left.\frac{1}{2} \tilde{\tau}_{a}\right|_{y=-d}=0 \text { lower surface }
\end{aligned}
$$

Note that the negative sign on the symmetric part of $\left.\tilde{\tau}\right|_{y=-d}$ is due to the sign convention defining the shear stresses on the upper and lower surfaces.

\section{Symmetric Solution}

The symmetric boundary conditions are

$$
\begin{array}{ll}
\left.\tilde{u}_{x}(\xi)\right|_{y=-d}=\left.\left.\tilde{u}_{x}(\xi)\right|_{y=-d} \quad \tilde{\tau}_{y x}(\xi)\right|_{y=-d}=-\left.\tilde{\tau}_{y x}(\xi)\right|_{y=d}=\frac{\tilde{\tau}_{a}}{2} \\
\left.\tilde{u}_{y}(\xi)\right|_{y=-d}=-\left.\left.\tilde{u}_{y}(\xi)\right|_{y=-d} \quad \tilde{\tau}_{y y}(\xi)\right|_{y=-d}=-\left.\tilde{\tau}_{y y}(\xi)\right|_{y=d}=0
\end{array}
$$

Because the motion is assumed symmetric, we retain only the constants $A_{2}$ and $B_{1}$ in Equation (17) and, upon substitution into (16), obtain

$$
\begin{gathered}
\tilde{u}_{x}=i \xi A_{2} \cos p y+q B_{1} \cos q y \\
\tilde{u}_{y}=p A_{2} \sin p y-i \xi B_{1} \sin q y \\
\tilde{\tau}_{y x}=\mu\left[-2 i \xi p A_{2} \sin p y+\left(\xi^{2}-q^{2}\right) B_{1} \sin q y\right] \\
\tilde{\tau}_{y y}=\mu\left[\left(\xi^{2}-q^{2}\right) A_{2} \cos p y+2 i \xi q B_{1} \cos q y\right] \\
\tilde{\varepsilon}_{x}=-\xi^{2} A_{2} \cos p y+i \xi q B_{1} \cos q y
\end{gathered}
$$

Substitution of Equations (20) and (21) into the boundary conditions (19) yields the linear system

$$
\begin{aligned}
& (-2 i \xi p \sin p d) A_{2}+\left[\left(\xi^{2}-q^{2}\right) \sin q d\right] B_{1}=\frac{1}{2 \mu} \tilde{\tau}_{a} \\
& {\left[\left(\xi^{2}-q^{2}\right) \cos p d\right] A_{2}+(2 i \xi q \cos q d) B_{1}=0}
\end{aligned}
$$

Solution of Equation (23) yields

$$
\begin{aligned}
A_{2}=\frac{\tilde{\tau}}{2 \mu} \frac{N_{A_{2}}}{D_{S}} \quad N_{A_{2}}= & 2 i \xi q \cos q d, \quad N_{B_{1}}=\left(\xi^{2}-q^{2}\right) \cos p d \\
B_{1}=\frac{\tilde{\tau}}{2 \mu} \frac{N_{B_{1}}}{D_{S}} \quad D_{S}= & \left(\xi^{2}-q^{2}\right)^{2} \cos p d \sin q d \\
& +4 \xi^{2} p q \sin p d \cos q d
\end{aligned}
$$

Note that the denominator $D_{S}$ is the same as the left-hand side of the characteristic equation (6). This indicates that the poles of Equation (24) correspond to the symmetric Lamb wave eigenvalues. Substitution of Equation (24) into Equation (22) yields the wavenumber domain displacement and strain at the plate upper surface

$$
\left.\tilde{u}_{x}^{S}\right|_{y=d}=-\left.\frac{1}{\xi} \frac{\tilde{\tau}}{2 \mu} \frac{N_{S}}{D_{S}} \quad \tilde{\varepsilon}_{x}^{S}\right|_{y=d}=-i \frac{\tilde{\tau}}{2 \mu} \frac{N_{S}}{D_{S}}
$$

where,

$$
N_{S}=\xi q\left(\xi^{2}+q^{2}\right) \cos p d \cos q d
$$

\section{Antisymmetric Solution}

The antisymmetric boundary conditions are

$$
\begin{aligned}
& \left.\tilde{u}_{x}(\xi)\right|_{y=-d}=-\left.\left.\tilde{u}_{x}(\xi)\right|_{y=-d} \quad \tilde{\tau}_{y x}(\xi)\right|_{y=-d}=\left.\tilde{\tau}_{y x}(\xi)\right|_{y=d}=\frac{\tilde{\tau}_{a}}{2} \\
& \left.\tilde{u}_{y}(\xi)\right|_{y=-d}=\left.\left.\tilde{u}_{y}(\xi)\right|_{y=-d} \quad \tilde{\tau}_{y y}(\xi)\right|_{y=-d}=-\left.\tilde{\tau}_{y y}(\xi)\right|_{y=d}=0
\end{aligned}
$$


Because the motion is assumed antisymmetric, we retain only the constants $A_{1}$ and $B_{2}$ in Equation (17) and, upon substitution into (16), obtain

$$
\begin{gathered}
\tilde{u}_{x}=i \xi A_{1} \sin p y-q B_{2} \sin q y \\
\tilde{u}_{y}=p A_{1} \cos p y-i \xi B_{2} \cos q y \\
\tilde{\tau}_{y x}=\mu\left[2 i \xi p A_{1} \cos p y+\left(\xi^{2}-q^{2}\right) B_{2} \cos q y\right] \\
\tilde{\tau}_{y y}=\mu\left[\left(\xi^{2}-q^{2}\right) A_{1} \sin p y+2 i \xi q B_{2} \sin q y\right] \\
\tilde{\varepsilon}_{x}=-\xi^{2} A_{1} \sin p y-i \xi q B_{2} \sin q y
\end{gathered}
$$

Substitution of Equations (28) and (29) into the boundary conditions (27) yields the linear system

$$
\begin{aligned}
& (2 i \xi p \sin p d) A_{1}+\left[\left(\xi^{2}-q^{2}\right) \cos q d\right] B_{2}=\frac{1}{2 \mu} \tilde{\tau}_{a} \\
& {\left[\left(\xi^{2}-q^{2}\right) \sin p d\right] A_{1}+(2 i \xi q \sin q d) B_{2}=0}
\end{aligned}
$$

Solution of Equation (31) yields

$$
\begin{aligned}
A_{1} & =\frac{\tilde{\tau}_{a}}{2 \mu} \frac{N_{A_{1}}}{D_{A}} \quad N_{A_{1}}=2 i \xi q \sin q d, \\
N_{B_{2}} & =-\left(\xi^{2}-q^{2}\right) \sin p d \\
B_{2} & =\frac{\tilde{\tau}_{a}}{2 \mu} \frac{N_{B_{2}}}{D_{A}} \\
D_{A} & =\left(\xi^{2}-q^{2}\right)^{2} \sin p d \cos q d+4 \xi^{2} p q \cos p d \sin q d
\end{aligned}
$$

Note that the denominator $D_{A}$ is the same as the lefthand side of the characteristic equation (7). This indicates that the poles of Equation (32) correspond to the antisymmetric Lamb wave eigenvalues. Substitution of Equation (32) into Equation (30) yields the wavenumber domain displacement and strain at the plate upper surface

$$
\left.\tilde{u}_{x}^{A}\right|_{y=d}=-\left.\frac{1}{\xi} \frac{\tilde{\tau}}{2 \mu} \frac{N_{A}}{D_{A}} \quad \tilde{\varepsilon}_{x}^{A}\right|_{y=d}=-i \frac{\tilde{\tau}}{2 \mu} \frac{N_{A}}{D_{A}}
$$

where,

$$
N_{A}=\xi q\left(\xi^{2}+q^{2}\right) \sin p d \sin q d
$$

\section{Complete Response}

The complete response to the PWAS excitation is obtained by combining the symmetric and antisymmetric responses, i.e.,

$$
\begin{aligned}
& \left.\tilde{\varepsilon}_{x}^{A}\right|_{y=d}=-i \frac{\tilde{\tau}_{a}}{2 \mu}\left(\frac{N_{S}}{D_{S}}+\frac{N_{A}}{D_{A}}\right) \\
& \left.\tilde{u}_{x}\right|_{y=d}=-\frac{1}{\xi} \frac{\tilde{\tau}_{a}}{2 \mu}\left(\frac{N_{S}}{D_{S}}+\frac{N_{A}}{D_{A}}\right)
\end{aligned}
$$

We now return from the wavenumber domain into the space domain by applying the inverse Fourier transform, i.e.,

$$
\begin{aligned}
\left.\varepsilon_{x}(x, t)\right|_{y=d}= & \frac{1}{2 \pi} \frac{-i}{2 \mu} \\
& \times \int_{-\infty}^{\infty}\left(\tilde{\tau}_{a}(\xi) \frac{N_{S}(\xi)}{D_{S}(\xi)}+\tilde{\tau}_{a}(\xi) \frac{N_{A}(\xi)}{D_{A}(\xi)}\right) e^{i(\xi x-\omega t)} d \xi \\
\left.u_{x}(x, t)\right|_{y=d}= & \frac{1}{2 \pi} \frac{-1}{2 \mu} \\
& \times \int_{-\infty}^{\infty} \frac{1}{\xi}\left(\tilde{\tau}_{a}(\xi) \frac{N_{S}(\xi)}{D_{S}(\xi)}+\tilde{\tau}_{a}(\xi) \frac{N_{A}(\xi)}{D_{A}(\xi)}\right) e^{i(\xi x-\omega t)} d \xi
\end{aligned}
$$

Though not explicitly shown, the functions $N_{S}, D_{S}, N_{A}$, $D_{A}$, also depend on $\omega$. The integral in Equation (37) is singular at the roots of $D_{S}$ and $D_{A}$, which are the symmetric and antisymmetric eigenvalues of the Rayleigh-Lamb equation, i.e., $\xi_{0}^{S}, \xi_{1}^{S}, \xi_{2}^{S}, \ldots$, and $\xi_{0}^{A}, \xi_{1}^{A}, \xi_{2}^{A}, \ldots$, Since the functions $D_{S}$ and $D_{A}$ depend on $\omega$, the number of eigenvalues that exist for a given $\omega$ will also vary. At low frequencies, i.e., $\omega \rightarrow 0$, only two eigenvalues exist, $\xi_{0}^{S}$ and $\xi_{0}^{A}$. At higher frequencies, several eigenvalues will exist. The evaluation of the integral in Equation (37) is done by the residue theorem, using a contour consisting of a semicircle in the upper half of the complex $\xi$ plane and the real axis (Figure 9). Hence:

$$
\begin{aligned}
\left.\varepsilon_{x}(x, t)\right|_{y=d}= & \frac{1}{2 \mu}\left(\sum_{\xi^{S}} \operatorname{Re}\left(@ \xi^{S}+\sum_{\xi^{A}} \operatorname{Re} @ @ \xi^{A}\right) e^{i(\xi x-\omega t)}\right. \\
= & \frac{1}{2 \mu}\left[\sum_{\xi^{S}} \tilde{\tau}_{a}\left(\xi^{S}\right) \frac{N_{S}\left(\xi^{S}\right)}{D_{S}^{\prime}\left(\xi^{S}\right)}+\sum_{\xi^{A}} \tilde{\tau}_{a}\left(\xi^{A}\right) \frac{N_{A}\left(\xi^{A}\right)}{D_{A}^{\prime}\left(\xi^{A}\right)}\right] \\
& \times e^{i(\xi x-\omega t)}
\end{aligned}
$$

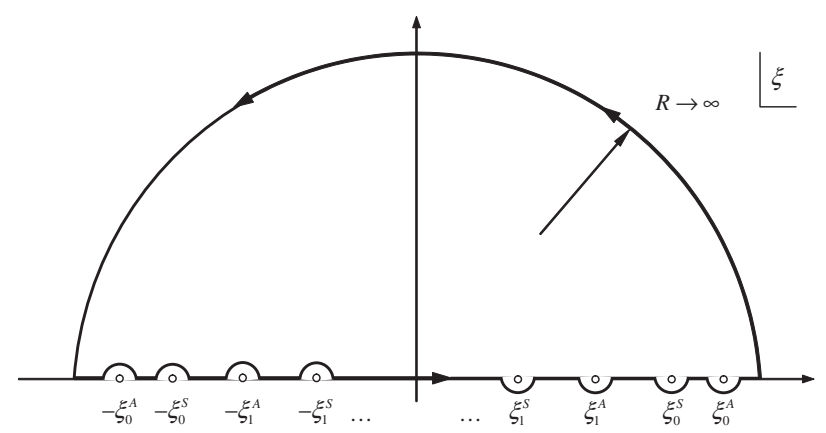

Figure 9. Contour for evaluating the inverse Fourier transform integral. Only the positive wave numbers are included. 
The summations are taken over all the symmetric and antisymmetric eigenvalues that exist at the particular frequency at which the analysis is conducted. Similarly,

$$
\begin{aligned}
\left.u_{x}(x, t)\right|_{y=d}= & \frac{-i}{2 \mu}\left[\sum_{\xi^{S}} \frac{\tilde{\tau}\left(\xi^{S}\right)}{\xi^{S}} \frac{N_{S}\left(\xi^{S}\right)}{D_{S}^{\prime}\left(\xi^{S}\right)}+\sum_{\xi^{A}} \frac{\tilde{\tau}\left(\xi^{A}\right)}{\xi^{A}} \frac{N_{A}\left(\xi^{A}\right)}{D_{A}^{\prime}\left(\xi^{A}\right)}\right] \\
& \times e^{i(\xi x-\omega t)}
\end{aligned}
$$

\section{Closed-form Solution for Ideal Bonding}

It was shown earlier that for thin and stiff bonding layers, ideal bonding between the PWAS and the structure may be asymptotically achieved. For ideal bonding conditions, the shear stress in the bonding layer takes the simple form of Equation (11), which admits a closed-form Fourier transform, i.e.,

$$
\begin{aligned}
\left.\tau(x)\right|_{y=d} & =a \tau_{0}[\delta(x-a)-\delta(x+a)] \\
\tilde{\tau} & =a \tau_{0}[-2 i \sin \xi a]
\end{aligned}
$$

Hence, the strain wave solution becomes:

$$
\begin{aligned}
\left.\varepsilon_{x}(x, t)\right|_{y=d}= & -i \frac{a \tau_{0}}{\mu} \sum_{\xi^{S}}\left(\sin \xi^{S} a\right) \frac{N_{S}\left(\xi^{S}\right)}{D_{S}^{\prime}\left(\xi^{S}\right)} e^{i\left(\xi^{S} x-\omega t\right)}-i \frac{a \tau_{0}}{\mu} \\
& \times \sum_{\xi^{A}}\left(\sin \xi^{A} a\right) \frac{N_{A}\left(\xi^{A}\right)}{D_{A}^{\prime}\left(\xi^{A}\right)} e^{i\left(\xi^{A} x-\omega t\right)}
\end{aligned}
$$

Similarly, the displacement wave solution becomes:

$$
\begin{aligned}
\left.u_{x}(x, t)\right|_{y=d}= & -\frac{a \tau_{0}}{\mu} \sum_{\xi^{S}} \frac{\sin \xi^{S} a}{\xi^{S}} \frac{N_{S}\left(\xi^{S}\right)}{D_{S}^{\prime}\left(\xi^{S}\right)} e^{i\left(\xi^{S} x-\omega t\right)}-\frac{a \tau_{0}}{\mu} \\
& \times \sum_{\xi^{A}} \frac{\sin \xi^{A} a}{\xi^{A}} \frac{N_{A}\left(\xi^{A}\right)}{D_{A}^{\prime}\left(\xi^{A}\right)} e^{i\left(\xi^{A} x-\omega t\right)}
\end{aligned}
$$

These expressions for Lamb wave response under PWAS excitation have been first published by Giurgiutiu (2003). Equations (41) and (42) contain the $\sin \xi a$ function. Thus, mode tuning is possible through the maxima and minima of the $\sin \xi a$ function. Maxima of $\sin \xi a$ occur when $\xi a=(2 n-1) \pi / 2$. Since $\xi=2 \pi / \lambda$, maxima will occur when the PWAS length $l_{a}=2 a$ equals on odd multiple of the half wavelength $\lambda / 2$. This is wavelength tuning. In the same time, minima of $\sin \xi a$ will occur when $\xi a=n \pi$, i.e., when the PWAS wavelength is a multiple of the wavelength. Since each Lamb wave mode has a different wavespeed and wavelength, such matching between the PWAS length and the wavelength multiples and submultiples will happen at different frequencies for different
Lamb modes. An extensive discussion of this effect in terms of wavenumber spectra was given by Sonti et al. (1995). Another factor that must be considered in Lamb wave tuning under PWAS excitation is the mode amplitude tuning, i.e., the matching of the PWAS location across the structural thickness with the modal displacement at that location. This factor is contained in the values taken by the function $N / D^{\prime}$. For example, if the PWAS is surface mounted, it is conceivable that, at a given frequency, some modes may have less surface amplitudes, while other may have larger surface amplitudes. Thus, two important factors for the design of PWAS-based Lamb wave embedded NDE for structural health monitoring have been identified:

(a) The variation of $|\sin \xi a|$ with frequency for each Lamb wave mode

(b) The variation of the surface strain with frequency for each Lamb wave mode

A plot of Equations (41) and (42) in the frequency range up to $1000 \mathrm{kHz}$ is presented in Figure 10. (The $S_{0}$ mode response is drawn with continuous line, while the $A_{0}$ mode response is drawn with dotted line.) It is apparent that the strain response is stronger at higher frequencies than the displacement response. This observation is consistent with the fact that the strain varies like $\sin \alpha$, while the displacement varies like $\operatorname{sinc} \alpha=\sin \alpha / \alpha$. This indicates that the PWAS transducers, which are strain coupled, may have a better high frequencies response than displacement and velocity transducers.

The Lamb wave mode tuning principles derived here for an exciter PWAS apply equally well for a receiver PWAS. In this case, the receiver PWAS would be able to selectively detect certain Lamb wave modes from a multimodal Lamb wave reception. An extension of this approach to circular crested Lamb waves was recently presented by Raghavan and Cesnik (2004).

\section{EXPERIMENTAL VERIFICATION OF PWAS GENERATED LAMB WAVES}

The basic principles of Lamb wave generation and detection by PWAS transducers were verified through simple laboratory experiments. A 1.6-mm thick, 2024aluminum alloy rectangular plate $\left(914 \times 504 \times 1.6 \mathrm{~mm}^{3}\right)$ was instrumented with eleven $7-\mathrm{mm}^{2}, 0.2-\mathrm{mm}$ thick PWAS (American Piezo Ceramics, APC-850) placed on a rectangular grid (Figure 11, Table 1). In this experiment, we verified that: (a) Lamb waves can be satisfactorily generated and detected with PWAS; (b) PWAS have an omnidirectional transmission; and 


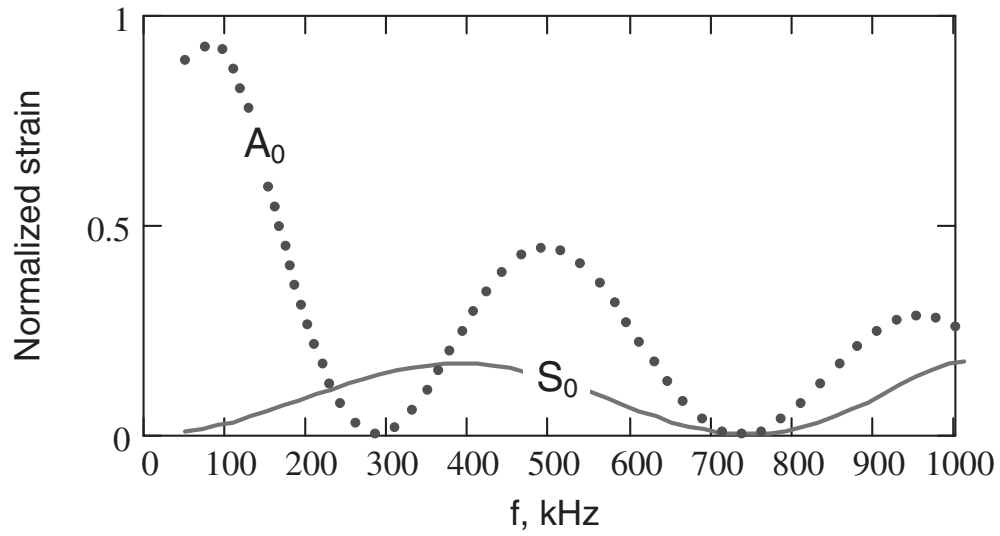

(a)

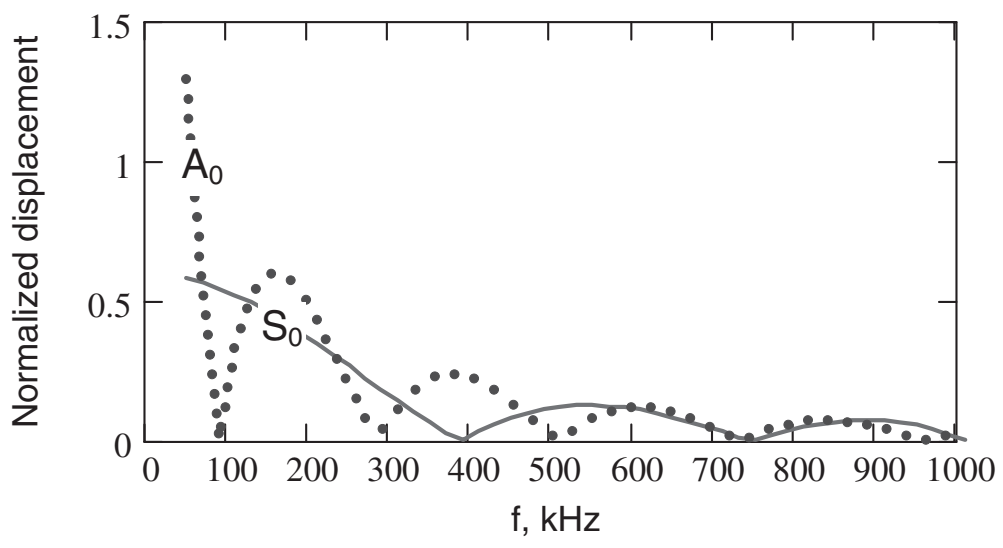

Figure 10. Predicted Lamb wave response of a 1.6$\mathrm{mm}$ aluminum plate under PWAS excitation: (a) strain response for a 7-mm PWAS; (b) displacement response for a 14-mm PWAS.

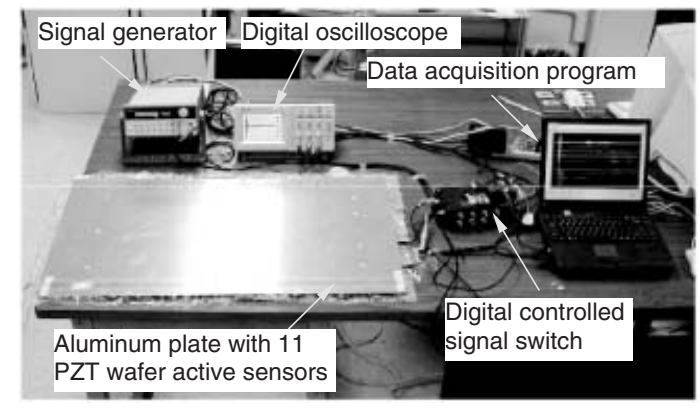

(a)

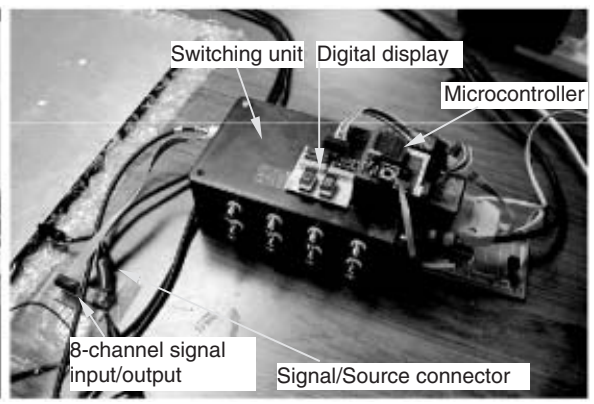

(b)

Figure 11. Experimental setup for rectangular plate wave propagation experiment: (a) overall view showing the plate, active sensors, and instrumentation and (b) detail of the microcontroller and switch box.

(c) signals are strong enough and attenuation is sufficiently low for echoes to be detected. The proof of these attributes is especially important for using PWAS in Lamb wave structural health monitoring, since PWAS are at least an order of magnitude smaller, lighter, and less power intensive than the conventional ultrasonic transducers.

Using a pair of PWAS placed at some distance apart, we verified first the mode tuning capabilities.
The transmitter was PWAS \#11 and the receiver was PWAS \#7. The distance between them was $760 \mathrm{~mm}$. The frequency was varied between 1 and $600 \mathrm{kHz}$. A three-count smoothed tone burst signal with a repetition rate of $16 \mathrm{~Hz}$ was generated with an $\mathrm{HP}$ $33120 \mathrm{~A}$ function generator and collected with a Tektronix TDS210 two-channel digital oscilloscope, synchronized with the signal generator (Figure 11). It was found that at low frequencies, the $A_{0}$ mode 
Table 1. Locations of sensors on the thin rectangular plate specimen.

\begin{tabular}{lccccccccccc}
\hline Sensor \# & $\mathbf{1}$ & $\mathbf{2}$ & $\mathbf{3}$ & $\mathbf{4}$ & $\mathbf{5}$ & $\mathbf{6}$ & $\mathbf{7}$ & $\mathbf{8}$ & $\mathbf{9}$ & $\mathbf{1 0}$ & $\mathbf{1 1}$ \\
\hline$x(\mathrm{~mm})$ & 100 & 100 & 100 & 100 & 100 & 450 & 450 & 450 & 800 & 800 & 800 \\
$y(\mathrm{~mm})$ & 100 & 175 & 250 & 325 & 400 & 100 & 250 & 400 & 100 & 250 & 400 \\
\hline
\end{tabular}

Table 2. Analysis of pulse echo signals of sensor \#11 on rectangular plate specimen.

\begin{tabular}{lcccccccc}
\hline $\begin{array}{l}\text { Wave Pack } \\
\text { Label }\end{array}$ & $\boldsymbol{R}_{\mathbf{1}}$ & $\boldsymbol{R}_{\mathbf{2}}$ & $\boldsymbol{R}_{\mathbf{3}}$ & $\boldsymbol{R}_{\mathbf{4}}$ & $\boldsymbol{R}_{\mathbf{5}}$ & $\boldsymbol{R}_{\mathbf{6}}$ & $\boldsymbol{R}_{\mathbf{7}}$ & $\boldsymbol{R}_{\mathbf{8}}$ \\
\hline $\begin{array}{l}\text { Time of flight } \\
(\mu \mathrm{s})\end{array}$ & 43.8 & 48.8 & 152.8 & 194.4 & 233.2 & 302.8 & 343.2 & 380.8 \\
$\begin{array}{c}\text { Path length } \\
(\mathrm{mm})\end{array}$ & 104 & 114 & 400 & 504 & 608 & 800 & 914 & 1008 \\
\hline
\end{tabular}

was excited very strongly while the $S_{0}$ mode was barely visible. As the frequency increased beyond $100 \mathrm{kHz}$, the $S_{0}$ mode gained strength, as shown in Figure 12(a). At frequencies beyond $200 \mathrm{kHz}$, the excitation of the $S_{0}$ mode increased dramatically while that of the $A_{0}$ mode diminished. At around $300 \mathrm{kHz}$, the excitation of the $S_{0}$ mode peaked, while the $A_{0}$ mode seamed to be completely rejected. Beyond $400 \mathrm{kHz}$, the $S_{0}$ mode became again very weak, while the $A_{0}$ mode picked up strength ${ }^{1}$. Thus, we have identified a 'sweet spot' for the preferential excitation of the $S_{0}$ mode at around $300 \mathrm{kHz}$. It must be noted that the excitation was performed solely with one surface mounted PWAS, i.e., placed asymmetrically about the midsurface of the plate. The tuning into the $S_{0}$ mode seemed to be entirely due to the wavelength matching effects that rejected the $A_{0}$ mode and encouraged the $S_{0}$ mode. In this frequency range, the across the thickness mode shapes of the $A_{0}$ and $S_{0}$ modes are such that maximum displacements are achieved at the plate upper and lower surfaces. Hence, no mode shape tuning was applicable. However, mode shape tuning will play a role at higher frequencies, where the mode shape distribution across the thickness changes considerably and $A_{1}, S_{1}$, and higher modes come into play (see Figure 3).

These experimental results were in very good agreement with the theoretical predictions. Figure 12(b) presents the theoretical prediction in roughly the same frequency range $(50-600 \mathrm{kHz})$. It can be seen that around $300 \mathrm{kHz}$, the $A_{0}$ mode goes through a minimum. This explains the $A_{0}$ rejection observed experimentally around this frequency. At the same time, the response of the $S_{0}$ mode at $300 \mathrm{kHz}$ is strong, and near its peak.

\footnotetext{
${ }^{1}$ Alas, $\mathrm{A}_{0}$ signal strength data beyond $450 \mathrm{kHz}$ were not recorded, since the focus of the research was on the tuning of the $S_{0}$ mode. Nevertheless, this recovery happened, as indicated by $\mathrm{A}_{0}$ group velocity data published by Giurgiutiu et al. (2003).
}

Thus, around this frequency, the excitation energy goes entirely into the $S_{0}$ mode. (Similarly, at around $100 \mathrm{kHz}$, the excitation energy was captured entirely in the $A_{0}$ mode.) Thus, we have shown that our predictions have been confirmed very well by the experiments.

\section{APPLICATIONS OF PWAS LAMB WAVES MODE TUNING TO STRUCTURAL HEALTH MONITORING}

The use of tuned Lamb wave modes in structural health monitoring is very important because it permits the researchers to address the detection of specific defects with specific Lamb wave modes. Finite element simulation studies performed by Giurgiutiu et al. (2003) indicated that $S_{0}$ waves get a much stronger echo from a through-the-thickness crack than $A_{0}$ waves. Thus the use of the pulse echo method for the detection of through-the-thickness crack is best performed with tuned $S_{0}$ wave. On the other hand, the $A_{0}$ waves seem more appropriate for the detection of disbond, delaminations, and corrosion with the pitch-catch method. While higher order Lamb wave modes, which manifest across-the-thickness nodes and maxima, may be best suited for the detection of internal defects that are placed at a depth that matches that of a particular Lamb mode. However, in this section we focus on the detection of through-the-thickness cracks in metallic plates using tuned $S_{0}$ Lamb wave mode and the pulse echo method.

First we verified that the PWAS can be used as both transmitter and receptor. Using the experimental setup of Figure 11, we sent a tuned $S_{0} 300 \mathrm{kHz}$ tone burst from PWAS \#11 and collected both the initial bang and the reflections from the plate edges. It was found that multiple reflections could be observed. It was also found that the tone burst wave packets maintain their shape even after multiple reflection, thus indicating that the $S_{0}$ waves have very little dispersion at this frequency. By processing the time of flight reflections, we concluded that strong echoes can be received from as far as $2500 \mathrm{~mm}$.

Next, we explored the use of a PWAS phased array and tuned $\mathrm{S}_{0}$ mode Lamb waves to create sweeping beams that can interrogate a large area from a single location. Real-time phased array systems have become very popular in NDE practice. Krautkramer (2002) produced a line of phased array transducers for the inspection of very thick specimens, and for the sidewise inspection of thick slabs, etc. with $P$-waves. For Rayleigh and Lamb waves, Deutsch et al. (1998a,b, 1999) developed a phased array self-focusing method, by which the delay time are adjusted to focus the beam exactly on the defect. The same concept could be used with embedded transducers, such as PWAS. Joshi (1995) 

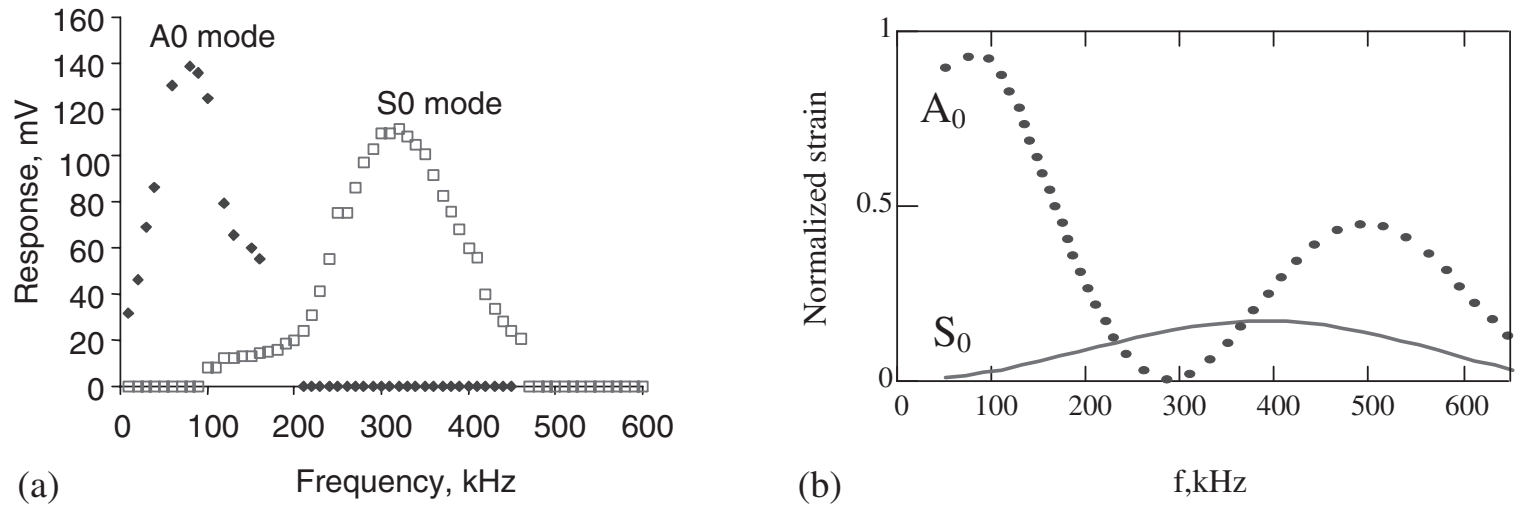

Figure 12. Experimental confirmation of the PWAS Lamb mode tuning capabilities: (a) excitation 'sweet spot' for $S_{0}$ mode observed experimentally at $300 \mathrm{kHz}$ in a 1.6- $\mathrm{mm}$ aluminum plate under a 7-mm PWAS excitation and (b) prediction of the $S_{0}$ excitation "sweet spot'.

used interdigital electrodes applied to a piezoelectric wafer to achieve Lamb wave excitation similar to that obtained with conventional comb transducers. Moulin et al. (2003) studied the feasibility of inducing beam steering with embedded rectangular PWAS. A threeelement array mounted on a composite plate was used as the transmitter of somehow directional beams of $S_{0}$ Lamb waves, while peripherally placed PWAS were used as receivers. Changes in the signal amplitudes due to damage were observed. Beam steering studies were also done by Purekar and Pines (2001, 2002), Sundaraman and Adams (2002, 2003), and others.

Our investigation led to the embedded ultrasonics structural radar (EUSR) concept (Giurgiutiu and Bao, 2002). This concept utilizes an array of closely spaced PWAS that are permanently attached to the structures and interrogate it at will. A full description of this concept was presented by Giurgiutiu and Bao (2002). This concept can be applied to various thin-wall structures such as aircraft, storage tanks, large pipes, etc. The EUSR concept was verified on an array of $N=9$ identical $7-\mathrm{mm}$ square PWAS placed at uniform $9-\mathrm{mm}$ pitch in the center of a $1220-\mathrm{mm}$ square aluminum plate (Figure 13(a)). The wave pattern generated by the phased array is the result of the superposition of the waves generated by each individual element. By sequentially firing the individual elements of an array transducer at slightly different times, the ultrasonic wave front can be focused or steered in a specific direction. Thus, sweeping and/or refocusing of the beam is achieved electronically without physically manipulating the transducers. To keep the instrumentation to a minimum, we adopted a virtual steering beam concept. In this concept, we use one PWAS as transmitter, but collect signals on all the PWAS. By applying a round robin routine, we switch the transmitter among all the PWAS in the array and collected a total number of $N^{2}$ elemental signals. This set of $N^{2}$ elemental signals is processed through the EUSR algorithm to generate a virtual sweeping beam that can be controlled from the front panel (Figure 13(b)). Once the beam steering and focusing was established, the detection of crack was done with the pulse echo method. During these experiments, the EUSR was used to detect cracks in two typical situations: (i) a $19-\mathrm{mm}$ broadside crack placed at $305 \mathrm{~mm}$ from the array in the $90^{\circ}$ direction; and (ii) a $19-\mathrm{mm}$ offdside crack placed at $409 \mathrm{~mm}$ from the array in the $136^{\circ}$ direction. Of these two, the latter was more challenging because the ultrasonic beam is not reflected back to the source but rather deflected sideways. Hence, the echo received from the offside crack is merely the backscatter signal generated at the crack tips. Figure 13(b) presents the front panel of the embedded ultrasonic structural radar graphical user interface (EUSR-GUI) created in LabView programming language. The signals being processed are those for the offside-crack. The virtual sweep is performed automatically and the half-plate image presented in the right pane is generated. Manual sweep can also be performed using the turn knob. The reconstructed A-scan signal for a particular beam angle is shown in the lower pane. In this case, the lower pane shows the signal reconstructed at the beam angle $\phi_{0}=136^{\circ}$, which corresponds to the crack location. We proved that inspection of a wide area is possible by creating a sweeping beam of ultrasonic Lamb waves that covered the whole plate from a central location.

\section{SUMMARY AND CONCLUSIONS}

This study has analyzed the mechanism through which piezoelectric wafer active sensors (PWAS) can excite and detect tuned Lamb waves. The capability of tuning certain Lamb wave modes is very important in structural health monitoring because defects respond differently to various Lamb wave modes. Thus, the detection of through-the-thickness cracks with the pulse 
(a)
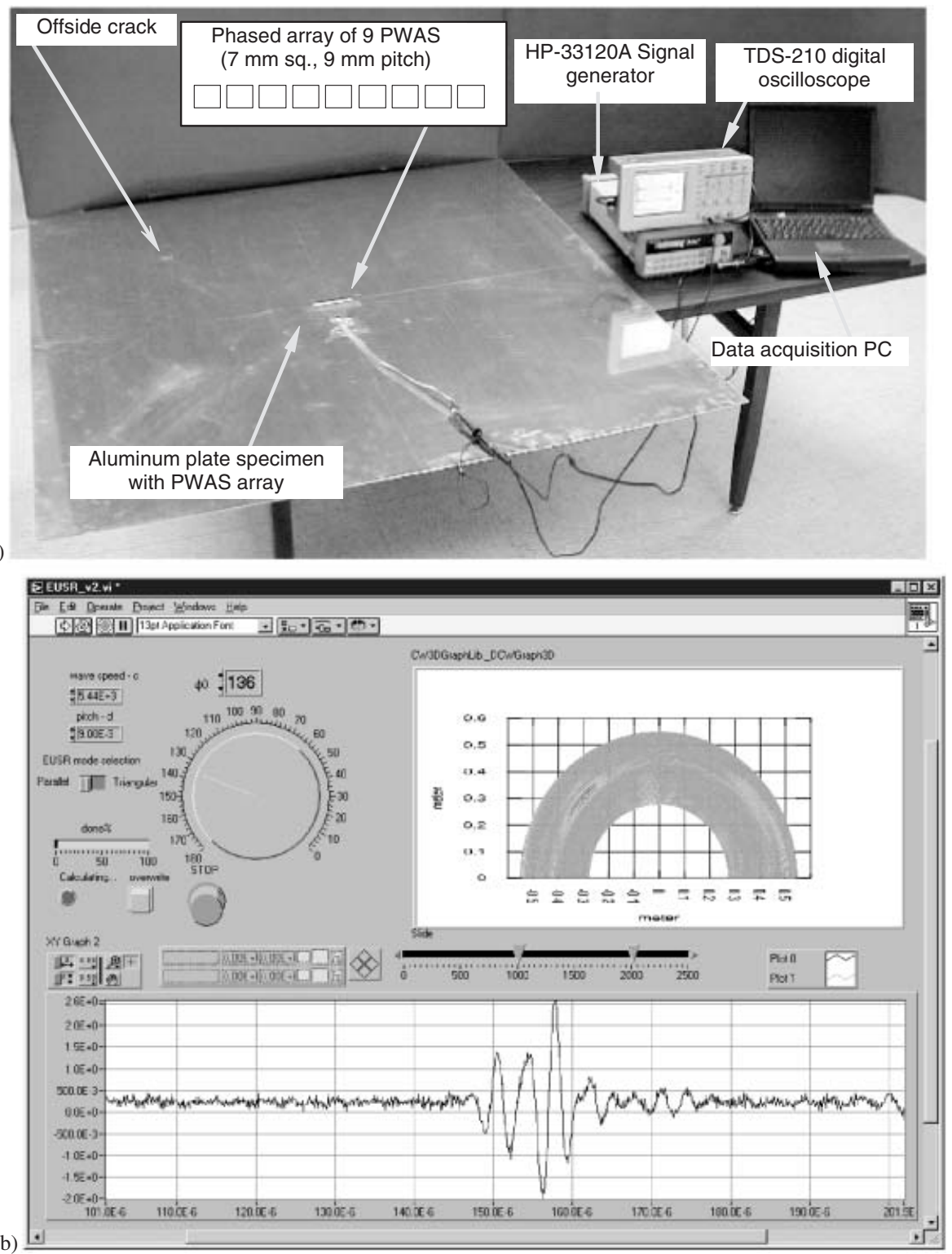

Figure 13. EUSR experiment: (a) thin plate specimen 9-element PWAS array and 19-mm offside crack and (b) GUI front panel showing the imaging of the structural defect at $\phi_{0}=136^{\circ}$ (offside crack). The corresponding echo is shown in the lower pane.

echo method is much better with the $S_{0}$ mode than with the $A_{0}$ mode. On the other hand, the $A_{0}$ mode seems to be better suited for the detection of delaminations, disbonds, and corrosion with the pitch-catch acoustoultrasonics techniques.

The analysis started with a brief review of the Lamb wave theory and of the fundamental equations. This was followed by an analysis of the PWAS operating principles and of the coupling between PWAS and structure through the adhesive layer. A model of the Lamb wave tuning mechanism with PWAS transducers was derived. The model was derived using the space domain Fourier transform. The analysis was performed in the wavenumber space. The inverse Fourier transform was used to return to the physical space. The integrals were evaluated with the residue theorem. A general solution was obtained for a generic expression of the interfacial shear stress distribution. The general solution was reduced to a closed-form expression for the case of ideal bonding which admits a closed-form Fourier transform of the interfacial shear stress distribution. It was shown that the strain wave response varies like $\sin \gamma a$, whereas the displacement response varies like sinc $\gamma a$. This indicates that PWAS transducers, which are strain coupled, are better suited for the excitation and detection of Lamb waves at high frequencies than the conventional ultrasonic transducers which are displacement coupled. Maximum excitation and 
detection are achieved when the PWAS length equals the half wavelength of a particular Lamb wave mode. Since Lamb wave modes wavelengths vary with frequency, the tuning of certain modes at certain frequencies can thus be achieved. Tuning curves were derived for the $A_{0}$ and $S_{0}$ modes. These curves were verified against experimental results at frequencies up to $600 \mathrm{kHz}$. A particular 'sweet spot' for the $S_{0}$ mode was found at $300 \mathrm{kHz}$ for a 7-mm PWAS attached to a 1-mm aluminum plate. Crack detection via the pulse echo technique using the phased array principle and tuned $S_{0}$ mode Lamb waves were demonstrated with the embedded ultrasonics structural radar (EUSR) algorithm. The sweeping of a large structural area from a central location and the successful detection of broadside and offside cracks in a 1200-mm square plate was demonstrated.

This study has shown that PWAS, in spite of their small size, are able to replicate many of the functions of the conventional ultrasonic transducers. PWAS are inexpensive, nonintrusive, unobtrusive, and minimally invasive devices that can be surface mounted on existing structures, or inserted between the layers of lap joints. PWAS can also be inserted between the layers of laminated composite materials. They can be placed inside closed cavities during fabrication/overhaul (such as wing structures or nuclear power plant piping), and then left in place for the life of the structure. PWAS are one of the enabling technologies for effective structural health monitoring. This emerging technology requires a sustained R\&D effort to achieve its full developmental potential for applicability to full-scale structural health monitoring systems.

\section{ACKNOWLEDGMENTS}

Support from the Air Force Research Lab through UTC Contract \#03-S470-033-C1 of F33615-01-D5801, National Science Foundation through grants NSF \#CMS-9908293 and NSF INT-9904493, Department of Energy through the Sandia National Laboratories contract doc. \# BF 0133 are thankfully acknowledged.

\section{REFERENCES}

Achenbach, J.D. 1999. Wave Propagation in Solids, North-Holland.

Alleyne, D.N., Pavlakovic, B., Lowe, M.J.S. and Cawley, P. 2001. "Rapid. Long Range Inspection of Chemical Plant Pipework Using Guided Waves," Review of Progress in QNDE, 20:180-187.

Agrawal, P. 1996. "Health Monitoring of Smart Structures by Method of Wave Scattering Techniques," MS Thesis, The University of Texas at Arlington, USA.

Butterworth-Hayes, P. 2003. "Europe Seeks 7E7 Work," In: Aerospace America, Nov. 2003, American Institute for Aeronautics and Astronautics, pp. 4-6.
Chang, F.-K. 1995. "Built-In Damage Diagnostics for Composite Structures," In: Proceedings of the 10th International Conference on Composite Structures (ICCM-10), August 14-18, 1995, Whistler, B.C., Canada, Vol. 5, pp. 283-289.

Chang, F.-K. 1998. "Manufacturing and Design of Built-in Diagnostics for Composite Structures," In: 52nd Meeting of the Society for Machinery Failure Prevention Technology, March 30-April 3, 1998, Virginia Beach, VA.

Crawley, E.F. and de Luis, J. 1987. "Use of Piezoelectric Actuators as Elements of Intelligent Structures," AIAA Journal, 25(10): 1373-1385.

Crawley E.F. and Anderson, E.H. 1990. "Detailed Models of Piezoceramic Actuation of Beams," Journal of Intelligent Material Systems and Structures, 1(1):4-25.

Dalton, R.P. Cawley, P. and Lowe, M.J.S. 2001. "The Potential of Guided Waves for Monitoring Large Areas of Metallic Aircraft Structure," Journal of Nondestructive Evaluation, 20:29-46.

Deutsch, W.A.K., Deutsch, K., Cheng, A. and Achenbach, J.D 1998. "Defect Detection with Rayleigh and Lamb Waves Generated by a Self-Focusing Phased Array," NDT.net, 3(12):1-6.

Deutsch, W.A.K., Cheng, A. and Achenbach, J.D. 1998. "Focusing of Rayleigh Waves: Simulation and Experiments," IEEE Transactions on Ultrasonics, Ferroelectrics, and Frequency Control, 46(2).

Diamanti, K., Hodgkinson, J.M. and Soutis, C. 2002 "Damage Detection of Composite Laminates Using PZT Generated Lamb Waves," 1st European Workshop on Structural Health Monitoring, July 10-12, 2002, Paris, France, pp. 398-405.

Diaz-Valdes, S.H. and Soutis, C. 2002 "Real-Time Nondestructive Evaluation of Fiber Composite Laminates Using Low-Frequency Lamb Waves," Journal of the Acoustical Society of America, 111(5):2026-2033.

Dupont, M., Osmont, R., Gouyon, R. and Balageas, D.L. 2000. "Permanent Monitoring of Damage Impacts by a Piezoelectric Sensor Based Integrated System", In: Structural Health Monitoring 2000, Technomic, pp. 561-570.

Giurgiutiu, V. and Bao, J. 2002. "Embedded Ultrasonic Structural Radar for the Nondestructive Evaluation of Thin-Wall Structures," In: Proceedings of the 2002 ASME International Mechanical Engineering Congress, November 17-22, 2002, New Orleans, LA, Paper \# IMECE2002-39017.

Giurgiutiu, V. and Zagrai, A. 2000. "Characterization of Piezoelectric Wafer Active Sensors," Journal of Intelligent Material Systems and Structures, 11(12):959-976.

Giurgiutiu, V., Zagrai, A.N., Bao, J., Redmond, J., Roach, D. and Rackow, K. 2002. "Active Sensors for Health Monitoring of Aging Aerospace Structures," International Journal of the Condition Monitoring and Diagnostic Engineering Management, 5(3).

Giurgiutiu, V., Bao, J. and Zhao, W. 2003. "PiezoelectricWafer Active-Sensor Embedded Ultrasonics in Beams and Plates," Experimental Mechanics, (December):428-449.

Graff, K.F. 1975. Wave Motions in Solids, Dover Publications, Inc.

Ihn, J.-B. and Chang, F.-K. 2002. "Built-in Diagnostics for Monitoring Crack Growth in Aircraft Structures," In: Proceedings of the SPIE's 9th International Symposium on Smart Structures and Materials, 17-21 March 2002, San Diego, CA, Paper \#4702-04.

Keilers, C.H. and Chang, F.-K. 1995. "Identifying Delamination in Composite Beam using Built-in Piezoelectrics," Journal of Intelligent Material Systems and Structures, 6:647-672.

Kim, Y.W., Varadan, V.V. and Varadan, V.K. 1997 "Modified Null Field Method for Elastic Wave Scattering from Partially Debonded Fiber, P and SV Waves," Composite Interfaces, 4, 1997.

Krautkramer 1998. "Emerging Technology - Guided Wave Ultrasonics," NDTnet, 3(6).

Krautkramer 2002, Products Catalog, http://www.krautkramer.com/ arrayweb/default.htm.

Lee, B.C. and Staszewski, W.J. 2003. "Modeling of Lamb Waves for Damage Detection in Metallic Structures: Part I. Wave 
Propagation; Part II. Wave Interaction with Damage," Smart Materials and Structures, 12:804-824.

Lin, X. and Yuan, F.G. 2001a. "Diagnostic Lamb Waves in an Integrated Piezoelectric Sensor/Actuator Plate: Analytical and Experimental Studies," Smart Materials and Structures, 10: 907-913.

Lin, X. and Yuan, F.G. 2001b. "Damage Detection of a Plate using Migration Technique", Journal of Intelligent Material Systems and Structures, 12(7).

Moulin, E., Assaad, J., Delebarre, C., Kaczmarek, H. and Balageas, D. 1997. "Piezoelectric Transducers Embedded in a Composite Plate: Application to Lamb Wave Generation," Journal of Applied Physics, 82:2049-2055.

Moulin, E., Bourasseau, N., Assaad, J. and Delebarre, C. 2003. "Lamb Wave Beam Steering for Integrated Health Monitoring Applications," SPIE, Vol. 5046, pp. 124-131.

Purekar, A.S. and Pines, D.J. 2001. "Interrogation of Beam and Plate Structures using Phased Array Concepts," In: Proc. of 12th ICAST, University of Maryland, College Park, MD, pp. 275-288.

Purekar, A.S. and Pines, D.J. 2002. "A Phased Sensor/Actuator Array for detecting damage in 2-D structures," In: Proc. of 43rd AIAA ASME/AHS/ASC Structures, Structural Dynamics, and Materials Conference, Denver, CO, Paper No. AIAA 2002-1547.

Raghavan, A. and Cesnik, C.E.S. 2004. "Modeling of Piezoelectricbased Lamb-wave Generation and Sensing for Structural Health Monitoring," SPIE, Vol. 5391, CD-ROM.

Rose, J.L. 1995. "Recent Advances in Guided Wave NDE," In: 1995 IEEE Ultrasonics Symposium, pp. 761-770.

Rose, J.L. 1999. Ultrasonic Waves in Solid Media, Cambridge University Press, New York.
Rose, J.L. 2002. "A Baseline and Vision of Ultrasonic Guided Wave Inspection Potential," ASME Journal of Pressure Vessel Technology - Special Issue on Nondestructive Characterization of Structural Materials, 124(3):273-282.

Seale, M.D., Smith, B.T. and Prosser, W.H. 1998. "Lamb Wave Assessment of Fatigue and Thermal Damage in Composites," Journal of the Acoustical Society of America, 103:2416-2424.

Silk, M.G. 1984. Ultrasonic Trancducers for Nondestructive Testing, Adam Hilger Publishers, ISBN 0852744366.

Sonti, V.R., Kim, S.J. and Jones, J.D. 1995. "Equivalent Forces and Wavenumber Spectra of Shaped Piezoelectric Actuators," Journal of Sound and Vibration, 187(1):111-131.

Sundaraman, S. and Adams, D.E. 2002. "Phased Transducer Arrays for Structural Diagnostics through Beamforming," In: Proc. of 17th American Society of Composites Technical Conference, West Lafayette, IN, CD-ROM paper.

Sundaraman, S., Adams, D.E. and Rigas, E.J. 2003. "Structural Damage Characterization through Beamforming with Phased Arrays," In: Proc. of the 4th International Workshop on Structural Health Monitoring, Stanford, CA, pp. 634-641.

Thomson, D.O. and Chimenti, D.E. (Editors) 2002. "Review of Progress in Quantitative Nondestructive Evaluation," Chapter $2 \mathrm{C}$ "Guided Waves" and Chapter 7 "NDE Applications," AIP Conference Proceedings, Vol. 615

Viktorov, I.A. 1967. Rayleigh and Lamb Waves, Plenum Press, New York.

Wang, C.S. and Chang, F.-K. 2000. "Built-In Diagnostics for Impact Damage Identification of Composite Structures," In: Chang Fu-Kuo (ed.), Structural Health Monitoring 2000, Technomic, Lancaster, PA, pp. 612-621. 